\title{
Die Behandlung der Keratoconjunctivitis eczematosa mit Partialantigenen nach Deycke-Much.
}

\author{
Von \\ Dr. Nikolaus Blatt. \\ Mit 3 Textabbildungen.
}

Die spezifische Therapie der tuberkulosen Erkrankungen - eine Zeitlang vernachlässigt, ja sogar von mancher Seite als schädlich verworfen - beginnt neuerdings wieder eine führende Rolle zu spielen. Auch bei Augenerkrankungen, die als tuberkulös angesehen werden, oder die - obzwar ihrer Atiologie nach unbekannt - mit irgendwelchen tuberkulösen Erkrankungen vergesellschaftet auftreten, beginnt die für spezifisch gehaltene Tuberkulintherapie allgemeine Anwendung zu finden. Man will die Lokalbehandlung des Auges durch die aktive Immunisierung des Organismus mittels des Tuberkelbacteriumextraktes unterstützen. Die unsichere und für giftig gehaltene Wirkung der älteren Präparate (Koch sches Alttuberkulin, Neutuberkulin, Bakterienemulsion) hatten zur Folge, daß eine ganze Reihe neuerer angegeben wurde, von denen das Partialantigenpräparat von DeyckeWuch das meiste Interesse erregte, dessen therapeutische Wirkung schon seit Jahren in der Literatur - pro oder contra - besprochen wird. Über die Anwendung der Partialantigene in der Ophthalmologie berichteten u. a. Kraupa, Köllner, Meyer, Schieck, insbesondere aber Nowak, der systematische Untersuchungen anstellte. Sie wurde bei den verschiedensten Augenerkrankungen erprobt, doch waren die Berichte über die Ergebnisse sehr divergierend und im allgemeinen nicht besonders günstig. Es gibt zwar Autoren, die gerade durch die Erfolge der Partialantigentherapie für die spezifische Behandlung gewonnen wurden, andere hingegen lehnten sie ab und verblieben bei der Tuberkulintherapie. Systematisohe, vergleichende Untersuchungen, an einem großen Material derselben Krankheitsgruppe angestellt, längere Beobachtung durch denselben Beobachter sind also nötig, um Klarheit zu schaffen.

Bei dem großen Umfange, den die Literatur über diese Frage in der letzten Zeit gewonnen hat, würde eine noch so flüchtige Wiedergabe derselben auf Schwierigkeiten stoßen. Es wird aber nötig sein, ganz 
kurz auf das Wesen der Partialantigenwirkung einzugehen, ehe ich meine eigenen Untersuchungen mitteile.

Die Anhänger dieser Therapie halten die Tuberkulinkur für ungenügend, weil die wirksamen Substanzen in den Tuberkulinpräparaten wenig aufgeschlossen. sind und auch im Organismus nicht hinreichend aufgeschlossen werden. - Dureh die Einwirkung der Milchsäure auf die Tuberkelbacillen dagegen werden die Leibessubstanzen derselben in die einzelnen wirksamen Bestandteile zerlegt. Weiterhin enthalten die Tuberkulinpräparate diese verschiedenen Bestandteile in schwankendem Mischungsverhältnis, daher liegt die richtige Dosierung jedes einzelnen derselben nicht in der Macht des Arztes. - Bei dem mittels der Milchsäure hergestellten Präparate dagegen lassen sich die einzelnen Bestandteile ganz voneinander separieren und können auch gesondert angewendet werden; so das Eiweißgemisch $=A$, das Fettsäurelipoidgemisch $=F$, das Tuberkulonastin $=N$. SchlieBlich enthäIt das Tuberkulin einen giftigen Stoff $(L)$, der durch die Milchsäure eliminiert werden kann. Es werden dann zur Therapie der Rest $(M T b R$ ) oder nur die einzelnen obenerwähnten Teile je nach Bedarf derselben benuitzt. Um die Art und die Menge der anzuwendenden Partialantigene zu bestimmen, muß die Art und die Menge der vorhandenen Antikörper bekannt sein (qualitative und quantitative Immunitätsanalyse). Dazu dient die Feststellung des Immunitätstiters, und zwar mittels Vergleichung der Lebhaftigkeit der Hautreaktion bei intracutaner Impfung der einzelnen Partialantigene. Je lebhafter die Realtion, desto größer die Menge des entsprechenden Antikörpers. Die Intensität der Reaktion mit den einzelnen Antigenen ist bei den verschiedenen Individuen verschieden (persönliches Immunitätsbild), und es muß dasjenige Antigen in gröBerer Menge angewendet werden, welches die geringere Reaktion gibt. Ist der Titer für alle drei Partialantigene gleich, so wird $M T b R$ benützt.

Meine Untersuchungen bezweckten die Feststellung des therapeutischen und diagnostischen Wertes der Deycke-Much schen Partialantigenpräparate bei dem unter dem Namen Keratoconjunctivitis eczematosa bekannten Krankheitsbilde.

Um zu einem objektiven Resultate zu gelangen, war außer der Verwendung eines genügend großen Krankenmateriales auch die Heranziehung anderer Behandlungsmethoden zum Vergleich nötig. Selbstverständlich dürfte die Lokalbehandlung in keinem Falle unterlassen werden. Ich teilte also die $150 \mathrm{Fälle}$, die ich beobachtete, in drei gleiche Gruppen, und behandelte die erste nur lokal, die zweite außerdem mit Tuberkulin, die dritte endlich außer der Lokalbehandlung mit Partialantigeninjektionen. Alle Fälle waren so ausgesucht, daß sie ein ungefähr gleichartiges Krankheitsbild und gleiche Prognose boten. Auch wurden meistens schwere, bereits seit längerer Zeit erkrankte Personen ausgewählt, die auch schon mehrere Rezidive durchgemacht hatten und mit anderen Behandlungsmethoden ohne dauernden Erfolg öfters behandelt waren. Die hiesige Umgegend bietet derartige Fäle in reichster Auswahl. Luetiker wurden ferngehalten, ebenso Fälle mit Hornhauterkrankungen, die nicht streng zum bekannten Bild der Keratoconjunctivitis eczematosa paßten, also nicht auf ekzematösskrofulöser Basis beruhten. Bei der Auswahl stützte ich mich haupt- 
sächlich auf das klinische Bild. Die probatorischen Injektionen wurden zwar angewendet, aber nicht als Maßstab benützt, da früher angestellte Untersuchungen in dieser Hinsicht ein sehr unsicheres Ergebnis zeigten. Das allgemeine Krankheitsbild der Patienten war selten das bekannte tuberkulöse; es ist ja, wie hinlänglich bekannt, die Vergesellschaftung dieser Keratitiden mit irgendwelchen tuberkulösen Erkrankungen durchaus nicht häufig. Dagegen waren bei sehr vielen der 150 Fälle Konstitutionsanomalien nachzuweisen. Teils gehörten sie zum quantitativ asthenischen Typus: Individuen von schwächlichem Körperbau, cyanotischer Hautfarbe, schwacher Muskulatur mit dünnen Knochen, Hypoplasie des Herzens und Gefäßsystems; teils zum qualitativ asthenischen: von starkem, pastösem Körperbau, mit blasser, fetter, schmieriger Haut, dickem Fettpolster. Beim ersteren Typus unterscheidet sich der Protoplasmaaufbau der Körperzellen nur quantitativ vom normalen, beim letzteren qualitativ. Die Unterscheidung dieser beiden Typen ist wichtig, da sich zwischen ihnen auch betreffs der therapeutischen Beeinflußbarkeit Verschiedenheiten ergaben, wie sich noch zeigen wird. Die Beschreibung des lokalen klinischen Bildes erscheint überflüssig, da ja dasselbe bekanntlich auch im Laufe derselben Erkrankung sehr wechselt, da weiterhin ein Zusammenhang desselben mit dem Erfolge der Therapie nicht nachzuweisen war. Nur mag noch einmal wiederholt werden, daß durchaus Fälle mit schweren Hornhautveränderungen, wie z. B. tiefen, zur Perforation neigenden Geschwüren, starken pannösen Auflagerungen, dickem GefäBband, starker, oberflächlicher Vascularisation, herangezogen wurden. Die Beobachteten waren im Alter zwischen 4 bis 20 Jahren, beiderlei Geschlechts, ohne daß Alter oder Geschlecht den therapeutischen Erfolg irgendwie beeinflußte. Die Beobachtung dauerte 3 Jahre, und zwar wurden die Patienten nach der lokalen Heilung im ersten Jahre 4 wöohentlich, später 8 wöchentlich untersucht. Trotz dex großen Mühe, die diese lange Beobachtungszeit bei der Indolenz und Unintelligenz vieler Kranken bereitete, mußte sie unbedingt durehgeführt werden, um über den sohlieBlichen Erfolg der angewendeten Heilmethode Aufschluß zu geben.

Bevor ich meine Erfahrungen mitteile, will ich noch einiges über die angewendete Technik mitteilen. Ich hielt mich in dieser Hinsicht an die Vorschriften von Deycke-Much und bin von ihnen nur bei den späteren Untersuchungen etwas abgewichen, wie es sich auf Grund der gewonnenen Erfahrungen als vorteilhaft erwies.

Die intracutanen, probatorischen Injektionen zur Festatellung des Immunitätsm titers wurden sowohl mit dem Gemisch $M T b R$ a ls auch mit den Antigenen $A-F-N$ angestellt, und zwar wurden folgende Lösungen verwendet: $A=1: 10$ Millionen, $1: 100$ Millionen, $1: 1000$ Millionen, $1: 10000$ Millionen; $F=1: 10000$ Millionen, $1: 100000,1: 1000000,1: 10$ Millionen; $N=1: 1000,1: 10000,1: 100000$, 
1 : 1000 000; zur Kontrolle wurde 0,5 proz. Carbolkochsalzlösung benützt. Alle Stiche wurden in einer Sitzung angelegt, die Ablesung geschah bei $A$ am vierten, bei $F$ und $N$ am siebenten Tage. Den Versuch einer Wiederholung der Probestiche habe ich bald aufgegeben, da die Änderungen des Intracutantiters einerseits keine strenge Gesetzmäßigkeit zeigten, andererseits für die richtige Dosierung keinen verwendbaren Wegweiser boten. Die meisten Autoren sind heute auch davon abgekommen, die Höhe der Anfangsdosis nach dem Ausfall des Immunitätstiters zu bestimmen, und man beginnt ganz allgemein mit den niedrigsten Dosen. Bei zu starken Reaktionen im Laufe der Therapie aber mußte die Einwirkung abgeschwächt werden, entweder, indem aie Injektionen zeitweilig eingestellt oder in längeren Zei vabschnitten gemacht wurden, oder endilich, indem man nicht weiter stieg, die eben erreichte Dosis wiederholte. Manche sahen bei diesem Verfahren nach weiterem Steigen Überempfindlichkeitserscheinungen. Bei dem hohen Grade der verwendeten Verdünnungen ist es wichtig, daß jeder Probestich mit einer besonderen Spritze und Kanüle gemacht werde, während dies bei den therapeutischen Injektionen nicht nöt:g ist. Von Deycke-Much ist zur Vereinfachung der Technik ein Instrumentarium angegeben worden, das aber kostspielig und leicht entbehrlich ist.

Die therapeutischen Injektionen werden am zweckmäßigsten mit kleinen Dosen, längere Zeit, mit langsamem Steigen gemacht. Und zwar ist es für den praktischen Arzt besonders vorteilhaft, wenn er sich aus den im Handel erhältljchen Stammlösungen $(M T b R=100000 ; F=1: 10000 ; N=1: 1000)$ je sechs Lösungen anfertigt, jede durch 10 fache Verdünnung der Vorangehenden. Es sind also von jedem Antigen mit der Stammlösung zusammen sieben Lösungen vorhanden (z. B.: MTbR 1 : 100 000, $1: 1000$ 000, 1 : 10 Millionen, 1 : 100 Millionen, 1 : 1000 Millionen, 1 : 10000 Millionen, 1 : 100000 Millionen).

Es wird nun immer mit $0,1 \mathrm{ccm}$ der schwächsten Lösung begonnen, bis $0,7 \mathrm{ccm}$ gestiegen und dann mit $0,1 \mathrm{~cm}$ der nächst höheren Lösung fortgesetzt. Das sind also zusammen 49 Dosen, die eine Kur ausmachen. Ob hier abgebrochen werden soll, hängt von den individuellen Verhältnissen ab. Es hat sich aber als vorteilhaft erwiesen, eine neue Kur nur nach Ablauf von 3 Monaten anzuschließen. Die Injektionen können täglich oder jeden 2. bis 3. Tag wiederholt werden. Das erstere soll vorteilhafter sein, da dadurch anaphylaktische Erscheinungen vermieden werden. Ich fand 2-3 tägliche Einspritzungen erfolgreicher. Zu den Injektionen benuitzte ich die Hinterflache des Oberarms, wo sie am wenigsten schmerzhaft waren. Nach den Injektionen traten Hautrötungen, Pusteln, in seltenen Fällen auch Allgemeinstörungen auf, die aber in bezug auf den therapeutischen Erfolg nicht verwertbar waren. Als auf kleine, aber wichtige Einzelheiten möchte ich auf das Schütteln der Lösung vor Gebrauch und auf die genaue Protokollierung hinweisen.

Schließlich noch einige Worte über die Tuberkulinbehandlung. Es wurde das Höchstsche Präparat „Koch I Alttuberkulin Nr. 44" verwendet, und zwar in den erwähnten Verdünnungen und mit derselben Technik, um einen Vergleich mit den Resultaten der P. A.-Therapie zu haben und eventuelle Verschiedenheiten nicht auf Verschiedenheiten der Technik beziehen zu müssen. Auch die diagnostischen Einspritzungen mit Tuberkulin bei der zweiten Gruppe habe ich nach dem Vorgang von Deycke-Much intracutan gemacht, was übrigens schon vor den genannten Autoren von Ellermann und Erlandsen versucht wurde. Die Ergebnisse waren aber dieselben, wie mit den Partialantigenen, d. h. nicht verwertbar.

Wie schon erwähnt, hatte ich ein doppeltes Ziel: erstens den diagnostischen und prognostischen, zweitens den therapeutischen Wert der Partialantigene festzustellen. Was das erste betrifft, so sind meine 
Erfahrungen, wie schon angedeutet wurde, durchaus ungünstig. Es wurde behauptet, daß der Ausfall der Immunitätsanalyse einen Wegweiser für die Art der anzuwendenden Antigene darstelle. Sind die Impfpapeln von $F$ und $N$ am stärksten, so können sowohl $A, F, N$ als auch $M T b R$ benützt werden; dagegen bei größerer Intensität der Reaktion mit $A$ nur $A+F+N$.

Ich hielt mich auch im Anfang an diese Regel, doch bald zeigte sich, daß sie der Erfahrung nicht standhielt und ich ging bald zu der am einfachsten durchzuführenden Behandllung mit $M T b R$ über, die übrigens auch Mülller für vorteilhaft hält, ohne irgendwelche Änderung des therapeutischen Erfolges. Auch eine Gruppierung im Hinblick auf die Prognose ließ sich auf Grund der Qualitätsverschiedenheiten der Analyse nicht durchführen.

Die Intensität der Reaktion soll auch zur Bestimmung der Anfangsdosis benützt werden können, wie schon erwähnt wurde. Dem ist aber auch nicht so; die Anstellung der Probe ist viel zu kompliziert, ihre Resultate viel zu unzuverlässig. - Dazu kommt noch, dab auf Grund dieser Probe viel zu oft eine zu hohe Anfangsdosis bestimmt wird, die bei weiterem Steigen dann zu einer Irritation führt. Es muß immer mit geringeren Konzentrationen angefangen werden, als dem Titer entspricht.

Die im Laufe der Therapie angestellten Proben sollen auch einen Prüfstein für die Leitung derselben, für thre Weiterführung oder Einstellung abgeben. Meine Erfahrungen widersprechen dem durchaus. Ich beobachtete Fälle mit negativer dynamischer Immunität (Müller), d. h. Fälle, deren Intracutantiter im Laufe der Therapie geringer wurde, die also zur Therapie ungeeignet schienen, bei denen trotzdem die weiter fortgeführte Therapie zu schönen Erfolgen führte. Im Gegensatz dazu standen Fälle mit positiver dynamischer Immunität, die der Therapie trotzten. Neben Fällen mit konstantem Intracutantiter und sehr labilem Krankheitsbilde waren solche da mit labilem Titer und konstantem Krankheitsbild. Übrigens hält auch Nowak die probatorischen Injektionen bei tuberkulösen Augenerkrankungen, sowohl im Hinblick auf die Diagnose als auch für die einzuleitende Behandlung für unzuverlässig, Much meint, daß „die Analyse selbstverständlich mit. der Prognose nichts zu tun hat". Damit stimmen auch meine Erfahrungen überein. Auch bei den lokal und mit Tuberkulin behandelten Fällen versuchte ich einigemal vor Beginn der Therapie die probatorischen Partialantigeninjektionen, doch ohne einen Wegweiser für den Erfolg der Therapie zu finden. Der Immunitätstiter und seine Änderungen scheinen durchaus labil zu sein und keinen Rückschluß auf die vorhandenen Abwehrkräfte des Organismus zu gestatten.

Im Gegensatz zu ihrer diagnostischen Verwendbarkeit haben sich 
mir die Partialantigene therapeutisch sehr wirksam erwiesen und waren den beiden anderen, zum Vergleich herbeigezogenen Methoden entschieden überlegen.

Was zunächst den Allgemeinzustand betrifft, so war die günstigere Einwirkung der Partialantigentherapie eklatant. Wir besitzen leider keinen absoluten Wertmesser zur Beurteilung dieser Einwirkung. Die Gewichtszunahme, die Müller als Maßstab empfiehlt, ist meiner Meinung nach als solcher nur bei der Gruppe der quantitativ asthenischen Kranken verwendbar. Jedenfalls gestattet sie dennoch einen gewissen Einblick in die Wirkung der Therapie, und auch von diesem Standpunkt ist die Überlegenheit der Partialantigenkur in die Augen springend, wie aus Skizze 1 ersichtlich. Sie zeigt das Verhältnis des Gesamtgewichtes der Patienten jeder Gruppe zu der Gewichtszanahme. Auch die eczematösen Nasenveränderungen sowie die verschiedenen Hauteruptionen heilten viel schneller ab. Bei manchen Kranken bestanden Beschwerden, die der zu Rate gezogene Internist auf Lungenspitzenprozesse bezog. Auch diese Beschwerden besserten sich im Laufe der spezifischen Therapie, und es konnte eine objektive Besserung des Prozesses nachgewiesen. werden. Noch wichtiger zur Beurteilung des Heilerfolges ist selbstverständlich die lokale Heilung. Leider besitzten wir in dieser Beziehung keinen

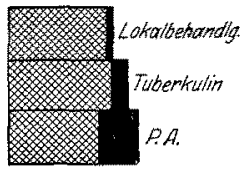

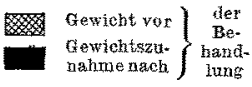
Abb. 1 . allgemein gültigen Maßstab. Das lokale Bild, der mehr oder minder sohnelle Ablauf der entzündlichen Erscheinungen läßt eine Orientierung zu, gestattet aber nicht die Aufstellung einer zahlenmäBigen Statistik. Jedenfalls war es zweifellos, daß die 100 mit Antigen behandelten Fälle sich viel schneller besserten, die entzündlichen Frscheinungen sich rascher abschwächten, die Aufklärung der Trübungen rascher vonstatten ging als die nur lokal behandelten (warme Umschläge, Atropin, Dionin, gelbe Salbe, Noviform, Jodol usw.). Manchmal traten Erscheinungen auf, die als Herdreaktionen zu deuten waren (Ciliarinjektion, Schmerzen, Dichterwerden der Trübungen). Daß aber die günstige Wirkung der Antigentherapie nicht etwa auf diesen Herdreaktionen beruhte, bewies der Umstand, daß sie durchaus nicht häufig waren und auch keine bessere Heilungstendenz schufen. Partialantigene und Tuberkulin wirkten im Hinblick auf dio Lokalheilung gleich gut.

Objektiver als der lokale Heilungsprozeß scheint als Maßstab der Heilwirkung die Sehschärfe zu sein. Doch ist zu bedenken, daß sie sehr von der Lokalisation des Krankheitsprozesses sowie von dem zeitlichen Einsetzen der Therapie abhängt, also ebenfalls kein absolutes Maß darstellt. Jedenfalls zeigte die nach 3jähriger Behandlungszeit angestellte Sehschärfeprüfung, daß der Visus der mit Antigen (Partial- 
antigen und Tuberkulin) Behandelten im allgemeinen ein besserer war als der Lokalbehandelten. Während bei den mit Antigen Behandelten die Zahl der Patienten mit fast normalem Visus ungefähr gleichgroß war, waren solche bei den Lokalbehandelten ijberhaupt nicht vorhanden.

Wie bekannt, besitzt die Keratoconjunctivitis eczematosa eine große Neigung zu Rezidiven, es ist also der Zusammenhang der Therapie mit der Stärke und besonders der Zahl der Rezidive von großer Wichtigkeit, um so mehr, als letztere auch einen zahlenmäßigen Beleg liefert. Auch in dieser Beziehung zeigte sich die Wirksamkeit der Partialantigene sehr schön und nicht nur der Lokal, sondern auch der

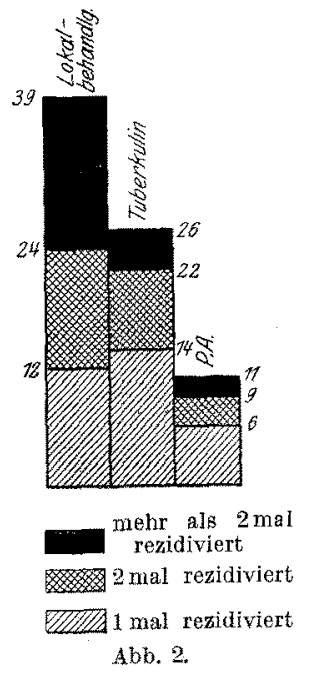
Tuberkulinbehandlung über-

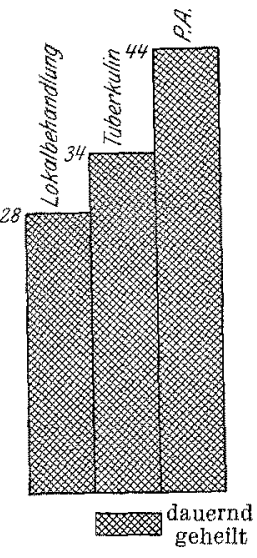

Abb. 3 . legen, wie Skizze 2 zeigt. Die Zahl der rezidivierten Erkrankungen war bei den zwei ersten Gruppen ungefähr gleich groß, nur die Anzahl der Rezidive bei den einzelnen Fällen war bei der Gruppe der mit Tuberkulin behandelten geringer. Die dritte Gruppe zeigte das günstigste Resultat, sowohl hinsichtlich der Gesamtzahl der Rezidive als auch der Häufigkeit derselben bei den einzelnen. Skizze 3 gibt Aufschluß über die Anzahl der dauernd Geheilten, die, wie ersichtlich, ebenfalls für die Partialantigentherapie spricht.

Wie lange die Behandlung fortgetührt, wann sie beendigt werden soll, muß immer nach den individuellen Verhältnissen bestimmt werden, eine allgemein gültige Regel gibt es nicht. Doch mahnen verschiedene Erscheinungen zur Einstellung der Therapie. So z. B. soll bei sehr lebhafter Infiltration der Injektionsstelle mit den weiteren Einspritzungen nur nach ihrer vollständigen Heilung fortgefahren werden. Ähnlich bei auftretenden hohen Temperaturen; während leichtere Temperatursteigerungen dies nicht begründen, da sie schnell vorübergehen.

Eine sehr große Wichtigkeit besitzt nach Deycke-Much die Höhe des Immunitätstiters. Da nach ihnen die Heilung in der Frhöhung dieses Titers begründet ist, so muß also mit der Therapie, und zwar nicht nur mit der Antigen, sondern auch mit anderen, z. B. Hochgebirge, Quarzlampe, Röntgen usw., so lange fortgefahren werden, bis 
der Titer eine genügende Höhe erreicht hat. Dies haben meine Untersuchungen nicht bestätigt, ja oft war das Entgegengesetzte der Fall; klinisch vollkommen geheilte Fälle ohne Rezidiv zeigten einen niedrigeren Titer. Umgekehrt war nicht selten bei nicht geheilten, hartnäckigen Fällen der Titer bedeutend erhöht. Es kann also als maßgebend für die Heilung nur das klinische Bild betrachtet werden. Zu diesen Erörterungen über die Dauer der Behandlung kann noch hinzugefügt werden, daß eine Kur nach 2-3 Monaten wiederholt werden kann.

Nachteile und Gefahren besitzt das Verfahren eigentlich keine. Dies bezieht sich übrigens auch auf die Tuberkulinbehandlung, wenigstens bei der Art, wie ich sie durchgeführt habe. Die Intracutaninjektionen auf der Hinterfläche des Oberarmes sind kaum schmerzhaft, die auftretenden kleinen Papeln dauern zwar ziemlich lange (8 bis 12 Wochen), heilen dann aber ohne Hinterlassung eines Zeichens. Sehr selten führten sie zur Entstehung von torpiden Eiterungen. Im Gegensatz zu den Partialantigeninjektionen führte das Tuberkulin manchmal zu schmerzhaften Hautreaktionen, aber nur bei höheren Dosen. Schwere Allgemeinsymptome waren nicht zu verzeichnen. Auch stärkere Herdreaktionen am Auge mit Verschlimmerung seines Zustandes traten weder bei der Partialantigen- noch bei der Tuberkulinbehandlung auf. Hochfiebernde Kranke, evtl. mit schweren Lungenprozessen, waren natürlich von der Antigentherapie ausgeschlossen.

Es wurde schon erwähnt, daß Fälle mit sogar leichteren Lungenprozessen selten waren. Dies wurde ganz exakt nachgewiesen, da alle Kranken von maßgebender Seite einer genauen, auch röntgenologischen Untersuchung unterzogen wurden. Auch die probatorischen Partialantigen- und Tuberkulininjektionen zeigten selten eine entschiedene Positivität. Eigentlich bedeutet eine positive Reaktion noch keine Erkrankung, sondern nur eine Überempfindlichkeit des Organismus, hervorgerufen durch die Anwesenheit von Tuberkelbacillen.

Das Schicksal des Organismus hängt aber nicht davon, sondern von der Körperbeschaffenheit ab. Dimmer hat auf diese auffallende Erscheinung, auf das Fehlen von tuberkulösen Erkrankungen bei der ekzematösen Keratitis aufmerksam gemacht. Ob dies ein Zufall ist, oder ob diese Keratitiden überhaupt nicht tuberkulöser Natur sind, sondern auf Grund einer hierzu disponierenden Körperbeschaffenheit durch verschiedene andere Ursachen hervorgerufen werden, diese Frage bedarf noch einer eingehenden Untersuchung. Es ist aber hier am Platz, auf die Ansichten einzugehen, die neuerdings über die Immunitätserscheinungen geäußert wurden, da sie für die Frage der Ätiologie und Therapie der ekzematösen Keratitis von Wichtigkeit sind.

Es wird angenommen, daß die sogenannte Immunität des Organismus schon ab ovo in der Konstitution gegeben ist, die die Wirkung aller unserer therapeutischen 
Maßnahmen von vornherein beeinflußt, in hemmendem oder förderndem Sinne. Daher die verschiedenen Hejlerfolge bei demselben therapeutischen Vorgehen und derselben Erkrankung bei verschiedenen Individuen. Mit dem angeborenen Gehalt des Organismus an Abwehrkräften ist es auch bei den ekzematösen Augenerkrankungen zu erklären, warum bei einem Kranken die Therapie versagt, während sie beim anderen vollen Erfolg hat. Diesen konstitutionellen Anteil stellt man sich vor als die angeborene Fähigkeit der Körperzellen, Abwehrstoffe zu erzeugen. Nur wenn diese Fähigkeit besteht, wenn diese Abwehrstoffe im Zellprotoplasma schon sozusagen latent vorhanden sind, kann die Therapie wirken, durch Aktivierung des Protoplasmas und Mobilisierung der Abwehrstoffe. Fehlt aber diese Fähigkeit, so bleibt die Therapie unwirksam. Ist die Zellentätigkeit nur quantitativ und nicht allzu stark herabgesetzt (quantitative Asthenie), so besteht die oben erwähnte Fähigkeit, die Therapie wird wirksam. Bei sehr starker quantitativer oder aber qualitativer Herabsetzung der Zellentätigkeit (qualitative Asthenie) ist diese Fähigkeit verloren gegangen, die Therapie bleibt ohne Erfolg, sei sie nun spezifisch oder unspezifisch.

Dadurch würde sich die Erscheinung erklären, warum bei meinen Kranken die mageren, abgeschwächten Individuen mit quantitativer Asthenie auf die Partialantigentherapie viel besser reagierten als diejenigen von pastösem Typus (qualitative Asthenie), die viel schwerer zu beeinflussen waren, öfters Rezidive bekamen und das größte Kontingent der Mißerfolge stellten. Übrigens war dies nicht nur bei den mit Partialantigenen, sondern auch bei den mit Tuberkulin, ja sogar bei den lokal behandelten Fällen zu konstatieren; bei allen fielen die Heilerfolge zugunsten der quantitativ Asthenischen aus. Der Erfolg der Partialantigenkur und der anderen Behandlungsmethoden ist nicht so sehr von einer kräftigen Antigenempfindlichkeit, $d . h$. von dem Immunitätstiter abhängig, als vielmehr von der Körperbeschaffenheit; von der noch vorhandenen Tätigkeit der Körperzellen (sowohl allgemein als auch lokal am erkrankten Herd), die durch das Partigenverfahren stärker angeregt wird. Übrigens war meistens die Immunitätsreaktion bei den quantitativ asthenischen Kindern intensiver.

Wollen wir die Wirkung der Partialantigentherapie und überhaupt der Antigentherapie mit der durch sie hervorgerufenen aktiven Immunisierung des Organismus erklären, so stoßen wir auf viele Schwierigkeiten. Daß bei der Antigentherapie das Wesen nicht in der Erzeugung von spezifischen, das Tuberkulosegift neutralisierenden Stoffen liegt, zeigten mir Augenerkrankungen, die mit Tuberkulose gar nichts zu tun hatten, und bei denen dennoch die Tuberkulin-, besonders aber die Partialantigeninjektionen einen Umschlag, ja eine Genesung der vorher allen Behandlungsmethoden trotzenden Erkrankungen herbeiführten. Daß die Tuberkulin- und Partialantigenwirkung nicht spezifisch ist, beweist auch die Tatsache, daß Fälle von Lungentuberkulose auf diese Weise sehr gut beeinflußbar sind, ohne daß im Sputum die bisher nachweisbaren Kochbacillen verschwunden wären. Weiter ist zu erwägen, daß die Wirkung des Tuberkulins und der Partialantigene 
nur vorübergehend ist; es heilt den momentanen Prozeß, sichert aber nicht unbedingt vor einer Reinfektion; eine Tatsache, die mit der Annahme einer aktiven spezifischen Immunisierung unvereinbar ist.

Daß das Tuberkulin nur auf lebendes, dagegen nicht auf abgestorbenes, verkästes Gewebe wirkt, deutet auch auf eine Protoplasmaaktivierung hin. Diese Aktivierung, diese Erhöhung der Zellentätigkeit kann auch durch Diät, Sonne, Röntgen, chemotherapeutisch erreicht werden, es kann also von einer spezifischen Wirkung gar nicht die Rede sein. Nach Bürger und Möller steht der exakte Nachweis der antigenen Eigenschaften der Tuberkelbacillenfette derzeit noch aus; auch im Tierversuch lösen die eiweißfreien Tuberkelbacillenfette keine spezifischen Reaktionen aus. Wäre die Tuberkulin- und Partialantigenwirkung spezifisch, so wäre es nicht möglich, daß die verschiedenartigsten Reize im Organismus ähnliche Reaktionen und Wirkungen hervorrufen. Schmidt fand ja, daß durch subcutane Injektion von $0,5 \mathrm{ccm}$ Milch bei aktiver Tuberkulose dieselben Stich-, Herdund Allgemeinreaktionen auftreten wie bei der Tuberkulinwirkung; auch die therapeutische Wirkung der Milchinjelstionen ist der therapeutischen Tuberkulinwirkung sehr ähnlich. Busacca ist es gelungen, beim Menschen durch intracutane Injektion von Pferdeserum bei aktiver Tuberkulose in $87 \%$ eine positive Reaktion zu erzielen. Herdreaktionen, wie man sie manchmal bei Tuberkulininjektionen findet, treten auch mit ganz andersartigen Eiweiskörpern auf. Wenn ich noch erwähne, daß die aktive, spezifische Immunisierung mit Tuberkulin im Tierversuch mißlang, so sind das, glaube ich, Tatsachen, die die Versuche zur Erklärung der Antigenwirkung auf eine, von der bisherigen verschiedene Weise begründen.

Darnach wäre die Antigenwirkung als ein artfremder biologischer Reiz aufzufassen. Dieser artfremde biologische Reiz - mag er auch Antigen genannt werden - erregt die Körperzellen zu einer lebhafteren biologischen Schutztätigkeit - mögen wir den Erfolg dieser Schutztätigkeit auch Antikörperbildung nennen. Es wäre also die Antigenwirkung der Proteinkörperwirkung ähnlich. Mit der Zeit werden wie dies Hayek meint - alle bisher auf verschiedene Art erklärten immunbiologischen Geschehnisse auf diese breitere Basis der biologischen Auffassung zurückzuführen sein. Auch die Wirkung der Partialantigene ist nur eine mittelbare, denn die Abwehrkräfte werden von den Körperzellen geliefert, welche durch die Partialantigene zu einer erhöhten Tätigkeit gereizt werden. Auf diese Weise können auch meine Ergebnisse erklärt werden, insbesondere das verschiedene Verhalten der quantitativ und qualitativ asthenischen, und die Tatsache, daß die Partialantigene insbesondere an der erkrankten Stelle (bei meinen Fällen an der Hornhaut) wirken, erklärt sich dadurch, daß die 
erkrankten, aber nicht abgestorbenen Zellen cine erhöhte Empfänglichkeit für den protoplasmaanregenden Reiz besitzen.

Es wäre noch zu erklären, warum die Partialantigentherapie wirksamer ist als die Tuberkulintherapie. Es ist wahrseheinlich, daß dies in der Gegenwart des giftigen Stoffes $L$ im Tuberkulin und in der Abwesenheit desselben in den Partialantigenen beruht. Es könnte durch die lange dauernde Anwendung des Tuberkulins zu einer Schädigung der Körperzellen führen, die die Empfindlichkeit derselben für die protoplasmaaktivierende Wirkung der Antigene herabsetzt. Die celluläre Wirkung der Antigene macht es auch erklärlich, warum intracutane Injektionen besser wirken als subcutane und intravenöse.

Zusammenfassend kann also gesagt werden, daß die therapeutische Anwendung der Partialantigene bei Keratoconjunctivitis eczematosa gute Exfolge gibt. Die Methode gehört um so mehr in die Hände des praktischen Arztes, als sie ambulant durchgeführt werden kann und den Patienten in seinem Berufe nicht stört. Insbesondere gilt dies von den geheilten Fällen, die zur Verhinderung von Rezidiven weiter behandelt werden. Auch die zweistïndigen Temperaturmessungen 3 Tage hindurch vor Beginn der Partialantigenkur ließen sich ambulant gut durchführen. Die Partialantigenkur ist für die ambulatorische Behandlung vorteilhafter als die Tuberkulinkur. Ein weiterer Vorteil ist, daß sie eine weitgehende Individualisierung gestattet. Daß die Heilwirkung nicht etwa auf einer Änderung der hygienischen Verhältnisse beruht, geht daraus hervor, daß die Kranken in ihrer bisherigen Um. gebung verblieben.

Im Gegensatz zur Therapie haben sich die Partialantigeninjektionen in der Diagnostik wenig bewährt. Die Partigenimmunitätsanalyse muß also sehr hypothetisch betrachtet werden.

\section{Literaturverzeichnis.}

Citron, Die Methoden der Immundiagnostik und Immuntherapie. - Deycke, Praktisches Lehrbuch der Tuberkulose. - Schild, Versuche als praktischer Arzt mit Partialantigenen bei chirurgischer und ähnlicher Tuberkulose. Berl. klin. Wochenschr. 1921, Nr. 6. - Landolt, Therapeutische Erfahrungen mit den DeyckeMuchschen Partialantigenen. Schweiz. med. Wochenschr. 1921, Nr. 18. - Dïll, Zur Behandlung der Lungentuberkulose mit deu Partialantigenen nach DeyckeMuch. Dtseh. med. Wochenschr. 1921, Nr. 32. - Schmidt, Zur Rehandlung chirurgischer Tuberkulose mit Partialantigenen. Dtsch. med. Wochenschr. 1920, Nr. 31. - Schulte-Tigges, Die Behandlung schwerer Fälle von Lungentuberkulose mit den Deycke-Muchschen Partialantigenen und die Bedeutung der Immunitätsanalyse bei der Behandlung derselben. Zeitschr. f. Tuberkul. 33, Heft 1. Miller, W., Partialantigene und Tuberkuline. Wien. klin. Wochenschr. 1917, Nr. 5. - Loewenhardt, Zur Behandlung der Tuberkulose mit Partialantigenen nach Deycke-Auch. Dtsch. med. Wochensohr. 1918, Nr. 48. - Ladwig, Erfahrungen mit Deycke-Muchschen Partialantigenen in der Behandlung der chirurgischen Tuberkulose. Bruns Beitr. z. klin. Chirurg. 119, Heft 3. - Klare, Der heutige 
Stand der Tuberkulosebehandlung mit Partialantigenen nach Deycke-Much. Intern. Zentralbl. f. Tuberkuloseforsch. 14, Nr. 1. 1920. - Deycke-Altstaedt, Weitere Erfahrungen in der Tuberkulosebehandlung mit Partialantigenen. Münch. med. Wochenschr. 1917, Nr. 9. - Müller, W., Untersuchungen über statische und dynamische Immunität bei Tuberkulose. Beitr. z. Klin. d. Tuberkul. 36. Müller, Neuere Anschauungen auf dem Gebiete des Lupus. Beitr. z. Klin. d. Tuberkul. 36. - Kögel, Die Beziehungen der Empfindlichkeit Tuberkulöser für Partialantigene. Beitr. z. Klin. d. Tuberkul. - Much, Tuberkulose. Ergebn. d. Hyg., Bakteriol., Immunitäts-Forsch. u. exp. Therapie 1917. - Hamburger und Peyrer Die negative und positive Phase der Tuberkulinempfindlichkeit. Wien, klin. Wochenschr. 1921, Nr. 14. - Hamburger, Über Tuberkuloseinfektion und Tuberkuloseerkrankung. Wien. klin. Wochenschr. 1921, Nr. 39. - Bürger und Möllers, Über den antigenen Charakter der Tuberkelbacillenfette. Wien. klin. Wochenschr. 1920, Nr. 37. - Hayek, Zur Proteinkörpertherapie. Wien. klin. Wochenschr. 1920, Nr. 35. - Gerber, Tuberkulinallergie und Lokalreaktion. Wien. klin. Wochenschr. 1920, Nr. 35. - Kirch, Ein Beitrag zur Tuberkulin. diagnostik der Lungentuberkulose. Wien. klin. Wochenschr. 1920, Nr. 35. Teilhaber, Die Verbesserung der Insuffizienz der Selbstscbutzvorrichtungen im Gewebe. Wien. klin. Wochenschr. 1920, Nr. 49. - Strassberg, Über eine neue Injektionsmethode des Tuberkulins bei avsgebreiteter Hauttuberkulose. Wien. klin. Wochenschr. 1922, Nr. 54 - Böhme, Über eine neue Injektionsmethode des Tuberkulins bei ausgebreiteter Hauttuberkulose. Wien. klin. Wochenschr. 1922, Nr. 8. - Busacca, Über eine neue Intracutanreaktion bei Hauttuberkulose. Wien. klin. Wochenschr. 1921, Nr. 47. -- Busson Die Proteinkörpertherapie und Vaccinbehandlung. Wien. klin. Wochenschr. 1922, Nr. 20. - Meller, Über die Behandlung von Augenkrankheiten mit Tuberkulin. Wien. klin. Wochenschr. 1922, Nr. 9. Nowak, Die spezifische Behandlung der Augentuberkulose durch den praktischen Arzt. Wien. klin. Wochenschr. 1922, Nr. 9. - Dimmer, Die Tuberkulose des Auges. Wien. klin. Wochenschr. 1922, Nr. 19. - Dietl, Abschwächung von Tuberkulin durch Tierkohle. Ned. Klinik 1921, Nr. 21. - Curschmann, Untersuchungen ïber Tuberkulinreaktionen. Med. Klinik 1921, Nr. 22. - Duschak, Herdreaktion und Prognose der Tuberkulinkur bei chirurgischer Tuberkulose. Med. Klinik 1921, Nr. 23. - Kämmerer, Über Tuberkulindiagnostik. Med. Klinilk 1921, Nr. 6-7. Möller, Über Prognosestellung bei der Lungentuberkulose mit Partialantigenen und Urochromogenreaktion. Med. Klinik 1921, Nr. 8. - Uhlenhut, Die experimentellen Grundlagen, der spezifischen Tuberkulosetherapie. Med. Klinik 1921, Nr. 24. Bauer, Konstitution und Tuberkulose. Med. Klinik 1921, Nr. 35. - Suess, Über Milchinjektionen bei Lungentuberkulose. Med. Klinik 1921, Nr. 46. - De la Camp, Die Strahlenbehandlung der Tuberkulose. Med. Klinil 1921, Nr. 48. - Schieck, Die Abhängigkeit des Verlaufs der tuberkulösen Prozesse am Auge von dem Stadium der Allergie des Gesamtorganismus. v. Graefes Arch. f. Ophthalmol. 105. 1921. - Igersheimer-Prinz, Gedanken und Untersuchungen zur Pathogenese der phlyktänulären Angenentzündungen und zum Schicksal skrofulöser Augenpatienten. v. Graefes Arch. f. Ophthalmol. 105. 1921. -Goerlitz, Tuberkulin bei Augenerkrankungen. Klin. Monatsbl. f. Augenheilk. 6\%. 1921. - Kraemer, Bemerkungen über die Allergie und Anergie bei Augentuberkulose. v. Graefes Arch. f. Ophthalmol. 10\%. 1922. - Nowak, Über die Partigenauswertung und Behandlung nach Deycke-Much bei tuberkulösen Erkrankungen des Auges. Verhandl. d. Ophthalmol. Gesellsch. in Wien 1921. - Nowak, Ergebnisse der Partigen behandlung Zeitschr. f. Augenheilk. 47. 1922. - Meyer, Die Behandlung skrofulöser Augenerkrankungen mit Partialantigenen nach Deycke-Much. v. Graefes Arch. f. Ophthalmol. 104. 1921. 